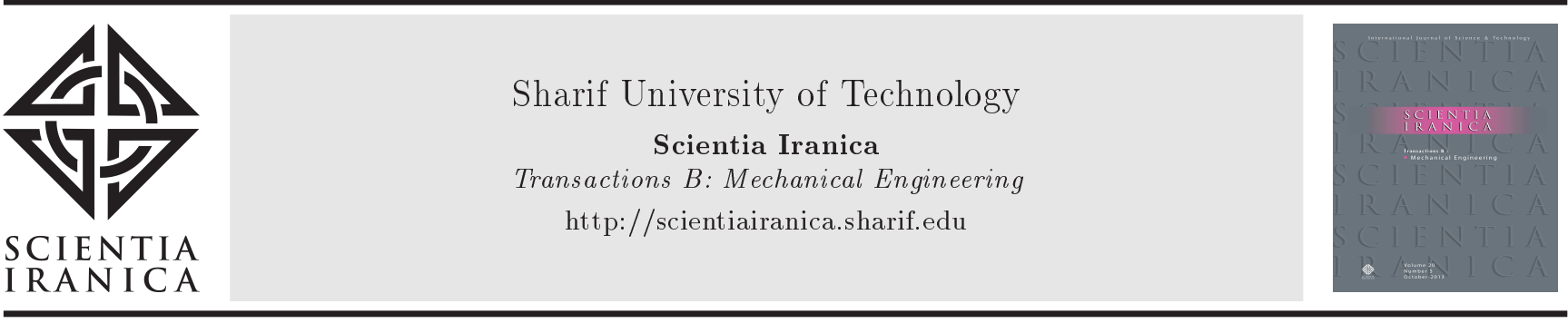

\title{
Multi-flute drill-broach for precision machining of holes
}

\author{
N. Dudak ${ }^{a}$, G. Itybayeva ${ }^{a}$, A. Kasenov ${ }^{a, *}$, Zh. Mussina ${ }^{a}$, A. Taskarina ${ }^{b}$, \\ and K. Abishev ${ }^{c}$ \\ a. Department of Mechanical Engineering and Standardization, S. Toraighyrov Pavlodar State University, Lomov Street 64, \\ Pavlodar, Kazakhstan. \\ b. Department of Metallurgy, S. Toraighyrov Pavlodar State University, Lomov Street 64, Pavlodar, Kazakhstan. \\ c. Department of Transport Engineering and Logistics, S. Toraighyrov Pavlodar State University, Lomov Street 64, Pavlodar, \\ Kazakhstan.
}

Received 20 November 2017; received in revised form 30 November 2017; accepted 12 February 2018

\author{
KEYWORDS \\ Multi-flute drilling \\ process; \\ The quality of \\ hole-making; \\ Hole-enlarging; \\ Hole surface \\ microstructure.
}

\begin{abstract}
This paper deals with hole-enlarging multi-flute drilling process. In this respect, the analysis of existing structures, their advantages and disadvantages is carried out and investigated. Cutting conditions during hole-enlarging multi-flute drilling process are shown. Herein, a new design of a hole-enlarging multi-flute drilling, as a holeenlarging multi-flute drill-broaching tool from broaching-speed steel with carbide plates, and a new way of handling new tools are presented. Hole-enlarging multi-flute drillbroaching combines the features of hole-enlarging multiflute drill (in cross-section) and those of broaching tool (in longitudinal section). In so doing, it is possible to increase the quality of hole-making (size variance, surface roughness) to facilitate cutting conditions and increase durability. The results of prototypes testing are presented in the end.
\end{abstract}

(C) 2019 Sharif University of Technology. All rights reserved.

\section{Introduction}

The main objective of machinery development is to improve products' quality constantly $[1,2]$. A way to do so is to increase the accuracy of surface treatment, which directly affects the functional quality of the product.

Optimization process of broaching and reaming tools is continuous and uninterrupted. In this respect, one can see some recent publications: a paper dedicated to geometrical optimization of broaching tool [3];

\footnotetext{
*. Corresponding author. Tel.: 8-77r7-435-06-35

E-mail addresses: nikolaydns@mail.ru (N. Dudak); galia-itibaeva@mail.ru (G. Itybayeva);

asylbek_kasenov@mail.ru (A.Kasenov);

mussina_zhanara@mail.ru (Zh. Mussina),

aya_taskarina@mail.ru (A. Taskarina);

a.kairatolla@mail.ru (K. Abishev)
}

doi: $10.24200 /$ sci. 2018.5623 .1379 articles dedicated to the analysis of the distribution of forces in the process of holes machining [4-6]; U.S. Patent of a new broaching tool, about which there are many new patents issued every year concerning new designs of tools, and this particular patent is only an example of such a continuous process [7,8]. Articles dedicated to the analysis of the relationship of the cutting forces with the resulting surface quality.

As is observed, holes machining is a branch in which the search and optimization of existing approaches continue and do not cease.

In the design of machine parts, one of the basic elements represents holes [9-11]. Depending on the requirements, the holes get drilling, reaming, unfolding, etc.

When countersinking, the cutting work is concentrated on a relatively small length of the cutting part, up to $15 \mathrm{~mm}$ (Figure 1). The cutting part is often perceived as high mechanical and thermal stresses, leading to wear increase, low resistance of 


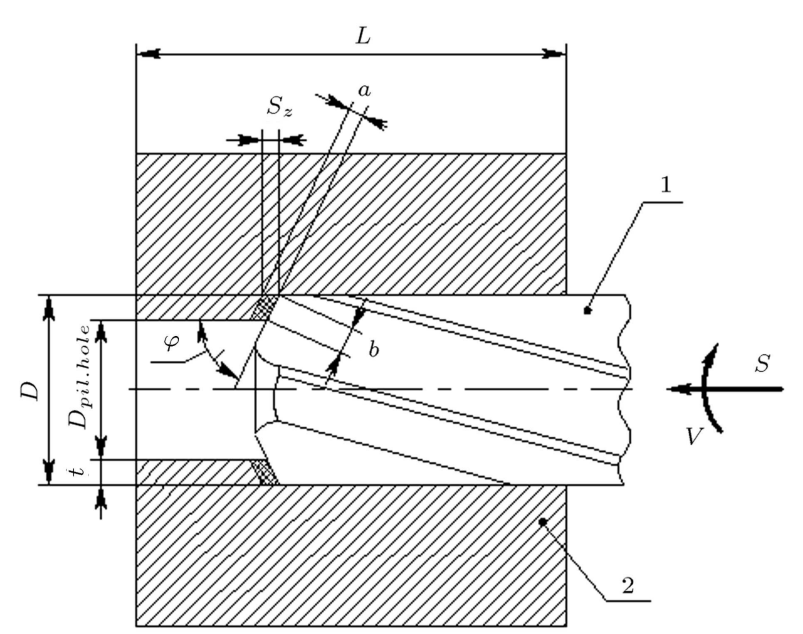

Figure 1. Elements of cutting at hole enlarging. 1: hole-enlarging multi-flute drill; 2: blank; $\varphi$ : the main angle in the plan; $a$ : thickness of cut; $b$ : width of cut; $t$ : depth of cutting; $D$ : diameter of the hole; $D_{o}$ : diameter of pilot hole; $L$ : length of treatment; $S$ : axis feed; $S_{z}$ : feed per tooth; and $V$ : speed of cutting.

hole-enlarging multi-flute drilling, and, consequently, low machining accuracy and surface roughness of the hole [12-14].

There are various hole-machining methods: honing operation, laser processing, and electro-chemical machining. Performing these methods requires expensive purchase of some equipment. Hole-machining operation by a multi-flute drill-broach tool is carried out on a universal vertically oriented drilling machine, which does not require equipment costs. It is available in any machine-building enterprise.

The main advantage of the hole-enlarging multiflute drilling is the ability to correct the hole axis and its shape.

Hole-enlarging multi-flute drilling process has a number of disadvantages: A high degree of the concentration of cutting edges at the hole-enlarging multiflute drilling provides cutting a large mass of metal [1517]. This, in turn, increases the concentration of forces and temperature of cutting in the tool body that deteriorates the conditions of its work. The growth of forces leads to increased roughness of the surface and, sometimes, to the damage of the instrument. The growth in temperature increases the wear of the tool.

In terms of eccentric compression, all the core tools are used for holes' machining work, including the hole-enlarging multi-flute drills. At the core tools, the shank is located behind the instrument $[18,19]$. The application of core tools is based on pushing through the holes leading to additional radial forces and to the breakdown of the processing holes diameter under the action of axial cutting force (Figure 2).

To illustrate this situation, a scheme of complex diagrams of specific acting forces (Figure 3) arising

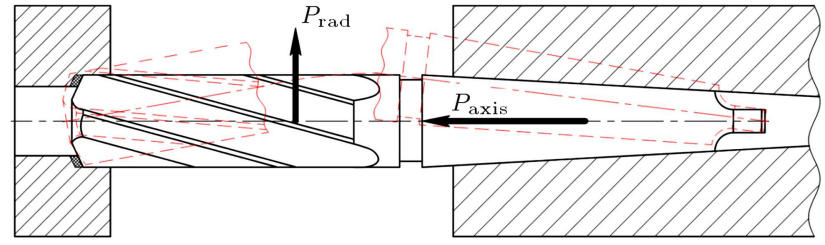

Figure 2. Scheme of axial force distribution on the cylinder of a standard hole-enlarging multi-flute drill.

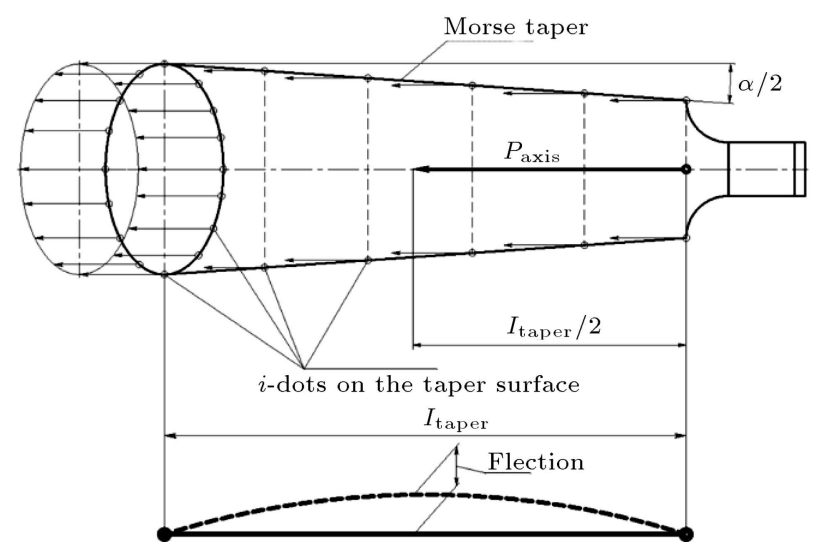

Figure 3. Scheme of acting specific forces complex diagrams.

from the action of axial cutting forces on the taper shank is shown.

$$
y=\frac{P_{\text {axis }}}{i},
$$

where $i$ is the number of selected points on the cone surface, varying from $n 1$ to $n 2$.

$$
P_{\text {axis }}=\sum_{i=n_{i}}^{n_{2}} P_{o y} \cdot i,
$$

where $P_{o y}$ is specific axial force acting on selected points, and $P_{\text {axis }}$ is axial force acting at the shank end.

Off-center compression and axis bending give rise to additional radial forces and to the breakdown of machined holes diameter. These effects also lead to the reduction of processing quality.

A cutting force arises when the broach teeth interact with the process material. It is divided into one acting along the direction of broach movement and into one perpendicular to such a direction.

In broaching, the round broach is under the stretching forces. Stretching force $P_{z}$ is opposite to the broach movement; stretching force $P_{y}$ is perpendicular to the broach movement (Figure 4). These forces $P_{z}$ and $P_{y}$ depend on the process material, cutting thickness, cutting length, the number of chip grooves on a tooth, and front and back angles.

The total force $P_{z}$ is composed of the specific forces $P_{z i}$, mutually equilibrated in action on the broach. 


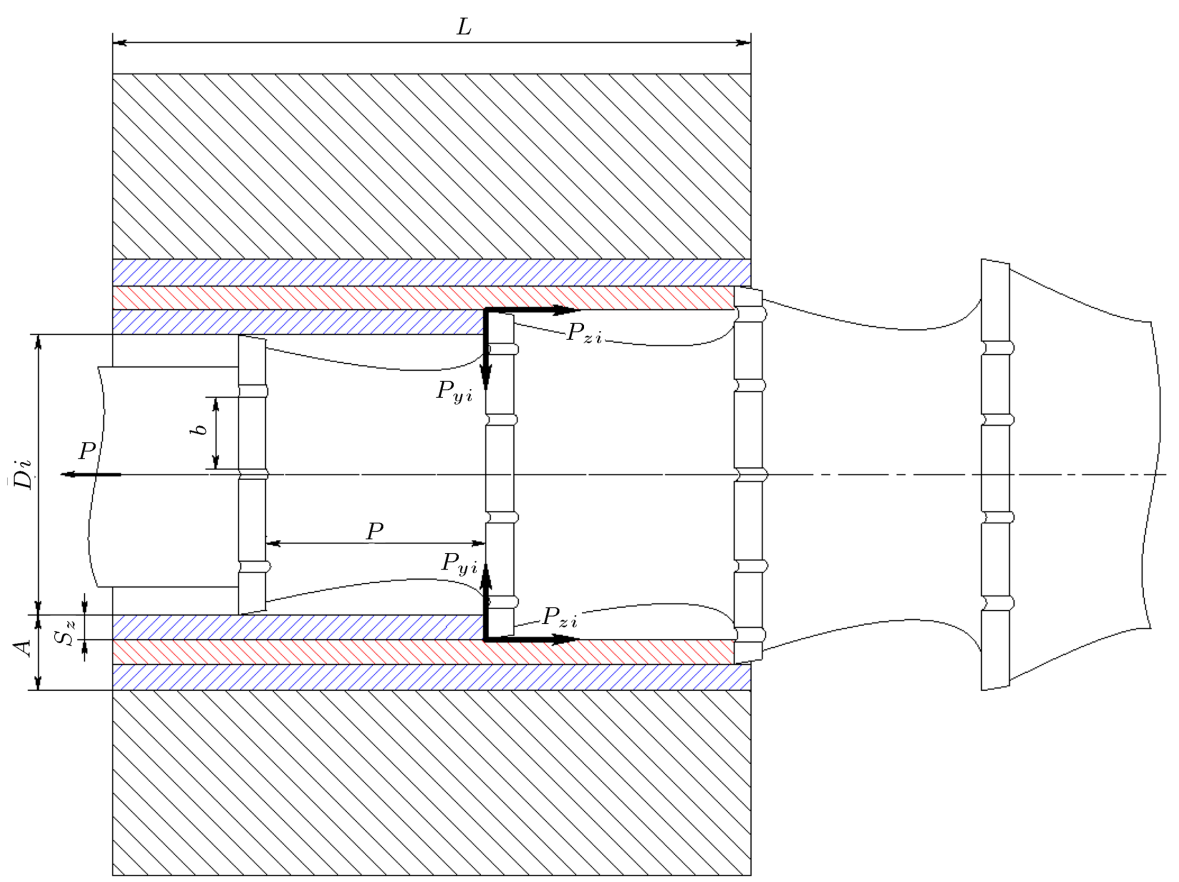

Figure 4. Scheme of forces acting in broaching. $D_{i}$ is broach teeth diameter; $L$ is length of broaching; $A$ is allowance; $S_{z}$ is advance per tooth; $b$ is cutting edge width; $p$ is tooth pitch; $P$ is broaching force; $P_{y i}$ and $P_{z i}$ are forces acting in broaching.

Based on $P_{z}$ component, necessary stretching force and the strength of broach elements (neck, crosssection along the first tooth) are determined. $P_{y}$ component squeezes the broach from the workpiece and affects accuracy in its size after broaching.

In broaching holes with round broaches, cutting force changes abruptly due to a variable number of simultaneously operating teeth, in which pitch is usually not a multiple of workpiece lengths. After the time $t$ equal to the ratio of cutting speed and teeth pitch $t=\frac{V}{p}$, the number of simultaneous operating teeth decreases by one tooth, cutting force decreases and the tooth, leaving the surface of a workpiece, stops (in our case, this is the tooth no. 1). In case the latter happens, the next tooth, engaging with the surface of a workpiece (in our case, this is the tooth no. 5), comes into operation (Figure 5).

After the tooth no. 1 leaves the surface of the workpiece, broaching force is reduced by $\frac{P}{z_{i}}$.

As a result, stress-strain state of a technological system is constantly changing. In addition, existing oscillations help reduce the surface quality (roughness and deviation from the specified geometric shape of a hole); moreover, broach resistance ability is reduced.

To eliminate the above-mentioned drawbacks, a new metal-cutting tool has been developed and proposed for machining holes, hole-enlarging multi-flute drilling broaching. The proposed tool combines the advantages of a drill, e.g., self-centering and alignment correction holes of complex profile and broach, and

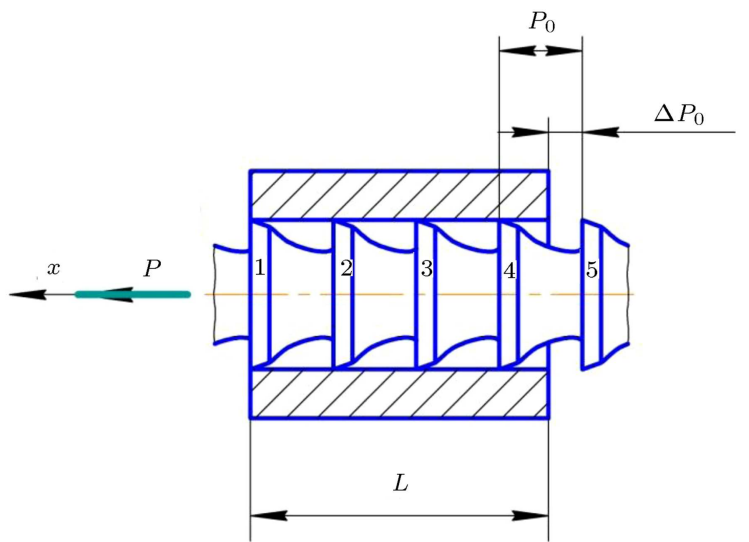

Figure 5. Scheme of broach teeth operation. $L$ is length of broaching, $P_{0}$ is tooth pitch; $\Delta P_{0}$ is distance between the workpiece and the next tooth no. 5 .

then, a favorable distribution of the forces in the body of the tool eliminates the beating and contributes to the high accuracy of the resulting hole. It provides improved cutting conditions, self-centring by dragging the tool through the hole, fixes shank front location, and increases the stability of the process.

Broach reamer is recommended to machine cylindrical holes of up to $40 \mathrm{~mm}$ in diameter and two diameters in length with accuracy rate about 6-7 points for the rough surface $R_{a}=0.08 \cdots 0.16 \mu \mathrm{m}$ and with allowance equal to the allowance for processing with reamers [20].

Hole-enlarging multi-flute drill-broach is recommended to machine cylindrical holes over $40 \mathrm{~mm}$ in 
diameter and up to two diameters in length in materials subjected to hardening with accuracy rate of about $7-8$ points for rough surface $\mathrm{Ra}=0.16, \cdots, 0.32 \mu \mathrm{m}$ and with an increased allowance up to $0.8-1.0 \mathrm{~mm}$.

Hole-enlarging multi-flute drill-broach is designed to remove more allowance in comparison with the broach reamer $\varphi_{1} \angle \varphi_{2}$, where $\varphi_{1}$ is cone angle of a broach reamer and $\varphi_{2}$ is cone angle of a hole-enlarging multi-flute drill.

\section{Method}

Methodological bases of research include dialectical and subject-activity approaches, methods of epistemological explication, conceptualization, methodological analysis, modeling, idealization, and structuralfunctional analysis.

To achieve this goal, methods of mathematical statistics and statistical modeling have been used. The experience of domestic and foreign scholars on the subject was summarized.

To verify the obtained results, an experiment was conducted, in which objects under study were delivered in a special, controlled and managed conditions. It gave an opportunity to consider the object of study and to obtain its full characterization.

Studies in this area began in 2008 and continued to date.

\subsection{Scheme of the hole-enlarging multi-flute drill-broach operation and description of the design}

The new cutting tool for machining holes is based on the combination of several types of operations in one, i.e., drilling and broaching. The use of new designs of reamers, called hole-enlarging multi-flute drills- broaches, eliminates negative factors of machining holes with existing hole-enlarging multi-flute drills and improves the quality and accuracy of holes machining and tool's life service.

Hole-enlarging multi-flute drill-broach, in which hole-enlarging multi-flute drill and broach properties combined in a single tool is designed to handle details such as sleeves, plugs, rings, etc. in the standard, largescale and mass production by 7 to 8 of IT Grade. Preliminary patents of the Republic of Kazakhstan have been received $[10,21,22]$.

Hole-enlarging multi-flute drill-broach is constructively built on the following principle; in axial section, it has structural features corresponding to the broach: front shank, neck, front and rear alignment, cutting and calibrated parts (they have helical teeth, and the cutting part has a conical shape, and the calibrating one has a cylindrical shape and extends through the hole as a broach) and, in a cross-sectional part, it has hole-enlarging multi-flute drilling features: the shape and number of teeth, the geometry of the cutting part (Figure 6); the proposed tool rotates during processing as the hole-enlarging multi-flute drill. Thus, the new tool is named hole-enlarging multi-flute drill-broach.

Figure 7 shows the cross-sectional profile and the shape of hole-enlarging multi-flute drill-broach teeth in the normal cross-sectional profile of cutting portion (versions 1, 2, 3) and of sizing part - version 4 . In the cross-section, the profile of hole-enlarging multi-flute drill-broach helical teeth can be of the following versions: standard profile of teeth (versions 2 and 3 ) and fixed width profile of teeth (version 1). Application of a fixed width profile of teeth will increase the resistance of hole-enlarging multi-flute drill-broach as well as the number of regrinding and, consequently, increase the

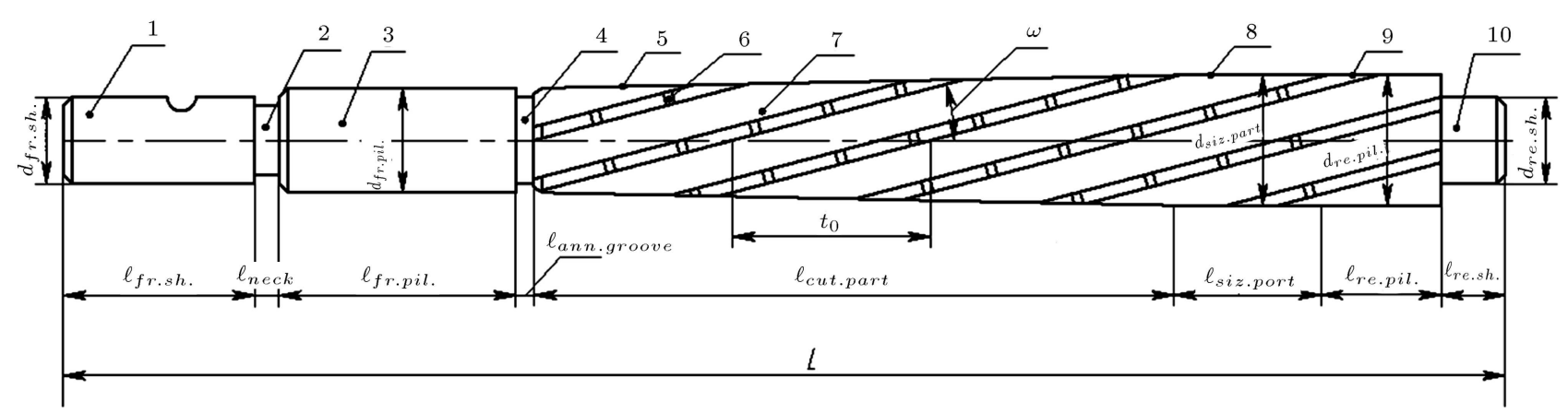

Figure 6. Structural elements of hole-enlarging multiflute drill-broach. $d_{f r . s h}$. and $d_{r e . s h}$ : diameters of the front and rear shank; $d_{f r . p i l}$ : diameter of the front pilot; $d_{s i z . p a r t}$ : diameter of the sizing part; $d_{\text {re.pil. }}$ : diameter of the rear pilot; $\ell_{f r . s h}$. and $\ell_{\text {re.sh. }}:$ lengths of the front and rear shank; $\ell_{\text {neck }}$ : neck length; $\ell_{f r . p i l}$. and $\ell_{\text {re.pil. }}$ : lengths of the front and rear pilot; $\ell_{\text {ann.groove. }}:$ length of the annular groove; $\ell_{\text {cut.part. }}:$ length of the cutting portion; $\ell_{\text {siz.part. }}:$ length of sizing part; $L:$ length of hole-enlarging multiflute drill-broach, $\omega$ : angle of spiral leads, direction like cutting direction; to: axial step of hole-enlarging multiflute drill-broach; 1: front shank; 2: neck; 3: front pilot; 4: an annular groove; 5: cutting portion; 6: chip-breaking groove; 7: clearance groove; 8: sizing part of hole-enlarging multiflute drill-broach; 9: rear pilot; and 10: rear shank. 


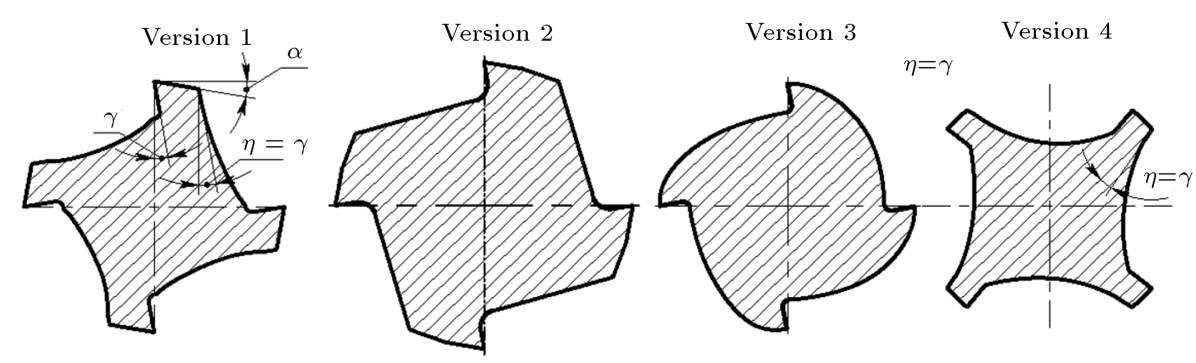

Figure 7. Cross-sectional profile of hole-enlarging multiflute drill-broach. $\gamma$ : front corner; $\alpha$ : back angle; and $\eta$ : the angle of the tooth back $(\eta=\gamma)$.
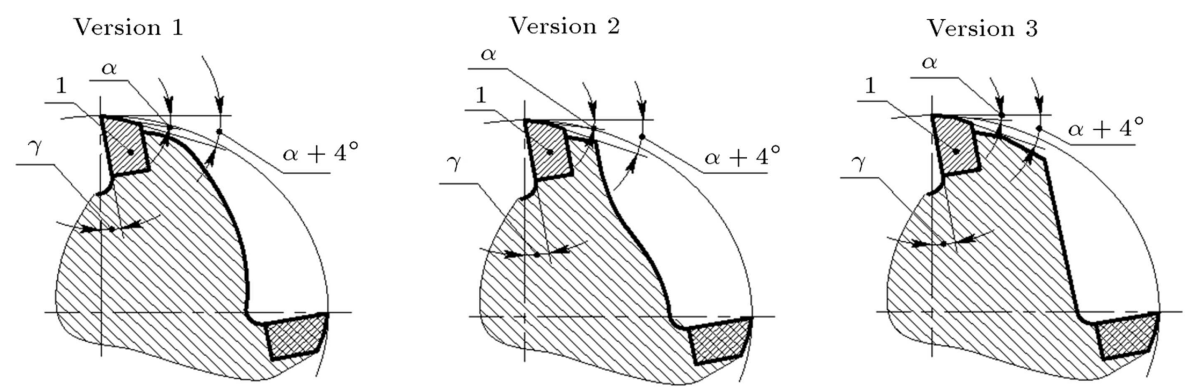

Figure 8. Cross-sectional profile of hole-enlarging multi-flute drill-broach with hard alloy plates. 1: hard alloy plate; $\gamma$ : front corner; and $\alpha$ : back angle.

service life due to regrinding flank surface, as opposed to the hole-enlarging multi-flute drill-broach with a round tooth compared with hole-enlarging multi-flute drill with a wire screw tooth, and recreate the state of the rear surface after regrinding to the state of a new instrument that will lead to higher quality of treatment. All the versions (1, 2, 3, and 4) can be refaced on the flank surface.

As a material for cutting, part of hole-enlarging multi-flute drill-broach can be as high speed as steel R6M5, R18, and soldered carbide plates. Crosssectional profile is presented in Figure 8.

Hole-enlarging multi-flute drill-broach concludes in its design elements of a hole-enlarging multi-flute drill and a broach, whose combination creates conditions that are more favorable for cutting. The cutting work is distributed along the long cutting part, as in broaching with a screw tooth, the shape of the teeth in the cross section with the profile of the chip groove, as well as working movements (a combination of rotational and translational movements)- as in the countersink. The design of hole-enlarging multi-flute drill-broach has such advantages of the broach as a relatively low cutting speed, processing quality (dimensional accuracy, surface roughness, and the reduction of abrasion), while the number of grooves as at the holeenlarging multi-flute drill from 2 to 6 depending on the diameter of the treatment.

Machinery parts' holes processing by the holeenlarging multi-flute drill-broach are performed on a lathe in the following ways: fixing hole-enlarging multi-

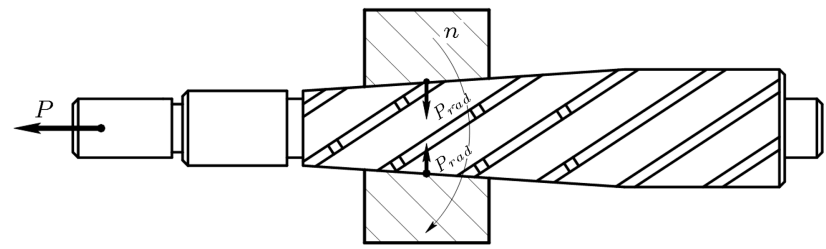

Figure 9. Distribution of forces by the holes processing with hole-enlarging multiflute drill-broach.

flute drill-broach in the cartridge or on the support of the lathe to the left or right direction depending on the direction of spindle rotation with the cutting tool.

In the design of the hole-enlarging multi-flute drill-broach, the shank is located at the front and the tool is pulled through the hole, eliminating breakdown of holes, followed by treatment with pushing the tool through the hole (Figure 9).

Thus, good centering of hole-enlarging multi-flute drill-broach and improvement of the quality of hole machining are achieved. In view of the above, the scheme of cylindrical holes processing is developed. At the front of the tool, the shank is located, eliminating the eccentric bending, because the hole-enlarging multi-flute drill-broach is pushed through the hole.

In the process of pushing with the hole-enlarging multi-flute drill-broach through the hole, this tool is centered, and its bending and axis withdrawal are excluded that improve the quality of holes processing. When machining by the proposed method, the tool is given a relative longitudinal movement as when pulling or the workpiece is given a longitudinal movement that 
relatively corresponds to pulling. Either hole-enlarging multi-flute drill-broach or workpiece is given a rotary motion inherent to the hole-enlarging multi-flute drill, which corresponds to reaming.

When processing with the hole-enlarging multiflute drill-broach, the following movements are used simultaneously:

1. A longitudinal movement along the axis (of workpiece or tool), which is inherent in broaching as the main cutting motion, denoted by $D_{r n}$;

2. Rotational motion inherent to reaming, denoted by $D_{r 3}$ (Figure 10).

When developing a new way, the synthesis of two processing methods has been performed:

1. Reaming with the main cutting motion $D_{r}$ by the rotation of hole-enlarging multi-flute drill or a part and axial feed of hole-enlarging multi-flute drill or a part $D$

2. Broaching with the main movement of cutting $D_{r}-$ translational motion of the tool and constructive feed $S_{z}$.

At the same time, in view of the constructive filing for hole-enlarging multi-flute drill-broach, feed movement of hole-enlarging multi-flute drill has become one of the main components of the resultant cutting motion. Constructive feed of hole-enlarging multi-flute drillbroach is done at the expense of the conical shape of working part with spiral teeth. With this arrangement, the supply is carried out continuously.

When processing with the hole-enlarging multiflute drill-broach, the feed motion of the tool is not available and is replaced by constructive feed for stock removal in the radial direction, as when broaching.

The teeth direction of the hole-enlarging multiflute drill-broach and a hole-enlarging multi-flute drill always coincide with the cutting direction and varies depending on the direction of rotation of the workpiece or tool during machining.
The combination of direct (right) rotational motion of the workpiece and translational motion of the hole-enlarging multi-flute drill-broach creates working conditions, and combination of the relative motion of the hole-enlarging multi-flute drill-broach and the workpiece, as during reaming holes, provides uniformity of teeth direction.

When machining by the proposed new method, the instrument is given a longitudinal movement or the workpiece is given a longitudinal displacement that corresponds to the broaching process. Rotary motion inherent in hole-enlarging multi-flute drill is given to hole-enlarging multi-flute drill-broach or to the workpiece that corresponds to reaming. The combination of rotational and translational motions is such that the tool always approaches the workpiece on the screw thread "ram" tooth from the obtuse angle between the cutting edges of the teeth and the axis of the hole-enlarging multiflute drill-broach; moreover, for the tool, the conditions for work of the hole-enlarging multi-flute drill and the hole-enlarging multi-flute drillbroach are enabled. The direction of helical teeth is similar to the cutting direction; a sectional shape of the chip groove (teeth) coincides with the shape of chip grooves of the hole-enlarging multi-flute drills; the number of teeth is in accordance with the requirements of the standard (Figure 11).

The axial broaching force value of hole-enlarging multi-flute drill is constant $P=$ const. The cutting process is accompanied by a rotational movement $M_{k}$ depending on the machining technique; bending moment $M_{u}$ appears due to drill axis bending.

Differential equation for hole machining with a hole-enlarging multi-flute drill-broach will be:

$$
m \frac{\partial^{2} x}{\partial t^{2}}+\mu \frac{\partial x}{\partial t}+c x+\frac{2\left(M_{k}+M_{u}\right)}{d}=F .
$$

The constant broaching force is an advantage of hole machining with helical broaches based on helical teeth $P=$ const. Consequently, force developed by operating cylinder is also constant, $F=$ const.

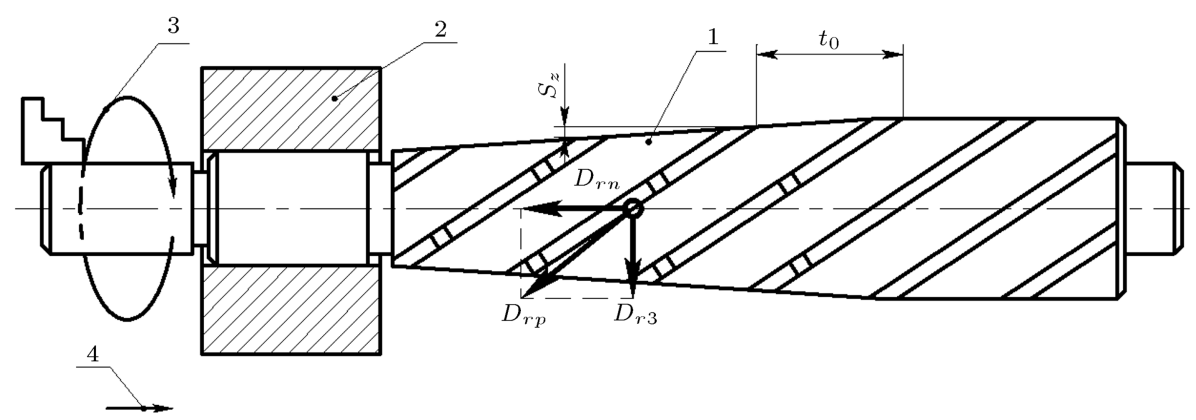

Figure 10. Scheme of the main cutting motions. 1: hole-enlarging multiflute drill-broach; 2: workpiece; 3: rotational motion in the designated point A gives the cutting motion inherent to reaming; 4: the progressive movement of the workpiece, causing the cutting force along the axis of the hole-enlarging multi-flute drill-broach and propelling the cutting inherent in broaching; $S_{z}$ : feed per tooth; $t_{o}$ : axial spacing between the teeth. 


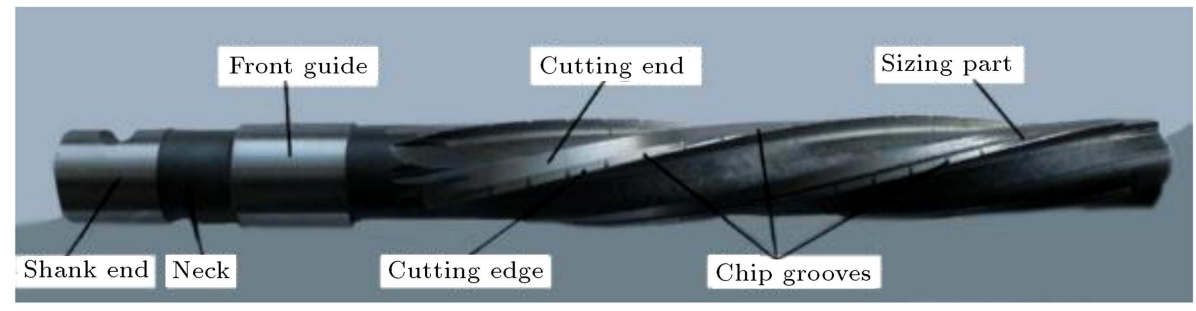

Fig ure 11. Hole-enlarging multiflute drill-broach.

$$
P=c x \text { : }
$$

Then the differential equation (3) will be as $F>$

$$
m \frac{\partial^{2} x}{\partial t^{2}}+\mu \frac{\partial x}{\partial t}+c x=F .
$$

Equation of motion of a point on the cutting edge of a broach with helical teeth shows that there must be tangential and additional axial forces due to helical teeth. Thus, a rotational moment takes place.

Then, the differential equation (4) will be as follows:

$$
m \frac{\partial^{2} x}{\partial t^{2}}+\mu \frac{\partial x}{\partial t}+c x+\frac{2 M_{k}}{d}=F
$$

where $d$ is the diameter of a broach, $M_{k}$ is the rotational moment, and $M_{k}$ has an additional effect on the broached workpiece.

In hole machining with a hole-enlarging multiflute drill-broach, axial force is equal to $P_{o c}$. It is lower than the force acting on the trip spindle of lathe $F, F>P$.

Force $P_{o c}$ is an additional axial force, performing thanks to helical teeth of a hole-enlarging multi-flute drill-broach.

If the rotational moment caused by helical teeth of a hole-enlarging multiflute drill-broach is taken into account, differential equation for machining with a hole-enlarging multiflute drill-broach countersinkbroach will be:

$$
m \frac{\partial^{2} x}{\partial t^{2}}+\mu \frac{\partial x}{\partial t}+c x=F-P_{o c} .
$$

The differential equation will be transformed, introducing the following notations $T_{k}=\sqrt{\frac{m}{c}} ; T_{g}=\frac{\mu}{c} ; K=\frac{1}{c}$; then:

$$
T_{k}^{2} \frac{\partial^{2} x}{\partial t^{2}}+T_{g} \frac{\partial x}{\partial t}+x=K .\left(F-P_{o c}\right) .
$$

Machining involves all the conditions for broaching holes with a helical broach.

To find the transient process for the case when $T_{g} \geq 2 T_{k}$, let us form a characteristic equation:

$$
T_{k}^{2} p^{2}+T_{g} p+1=0 .
$$

Its solution is in the following:

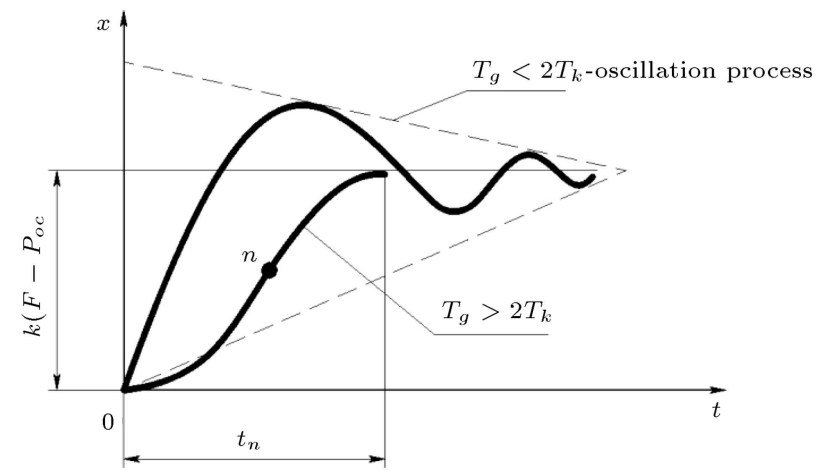

Figure 12. Graph of transient process.

$$
P_{1,2}=\frac{-T_{g} \pm \sqrt{T_{k}^{2}-4 T_{k}^{2}}}{2 T_{k}^{2}} .
$$

The general integral for $T_{g}>2 T_{k}$ is:

$$
x_{n}=c_{1} e^{-p_{1} t}+c_{2} e^{-p_{2} t} .
$$

The steady-state value is $x_{y}=K\left(F-P_{o c}\right)$, because $\frac{\partial x}{\partial t}=0$ and $\frac{\partial^{2} x}{\partial t^{2}}=0$.

The complete solution to the differential equation:

$$
x=x_{n}+x_{y}=C_{1} e^{-P_{1} t}+K\left(F-P_{o c}\right) .
$$

Integration constants $C_{1}$ and $C_{2}$ are determined conventionally from initial conditions when $t=0 ; x=0$; and $\frac{\partial x}{\partial t}=0$.

The graph of transient process consists of two exponents with a flex point $n$ (Figure 12).

The nature of transient processes and their duration depends on values $T_{k}$ and $T_{g}$ that include the system parameters.

Unlike the hole enlarging with a standard drill, the same process with a hole-enlarging multi-flute drillbroach is more stable, because a drill is self-aligning in the hole during the "broaching". Equation solution shows that the system is stable, as can be seen from the graphs of transient process.

The developed mathematical model, describing the process of hole machining with a hole-enlarging multi-flute drill-broach, allows the calculation of the system parameters to solve the problems of mechanical design and machining technologies. 


\subsection{Experimental studies on hole-enlarging multiflute drill-broach processing}

Hole machining was carried out on the screw-cutting lathe of model 1A616, and geometric accuracy assessment was carried out in accordance with the GOST standard 18097-93 by the Euro-Asian Council for Standardization, Metrology and Certification (EASC). For the experiments, samples of parts were prepared. The samples were drilled with a diameter equal to that of the front rail of the hole-enlarging multi-flute drillbroach (Figures 13 and 14).

Processing with hole-enlarging multi-flute drillbroach was done using cooling lubricants. When processing the steel, a $10 \%$ solution of the emulsion was used as a lubricating and cooling liquid; in the treatment of bronze and iron, a $10 \%$ solution of the emulsion and kerosene was used. Adding kerosene is a common practice used in drilling, which provides high drilling rates and a reduction in differential sticking.

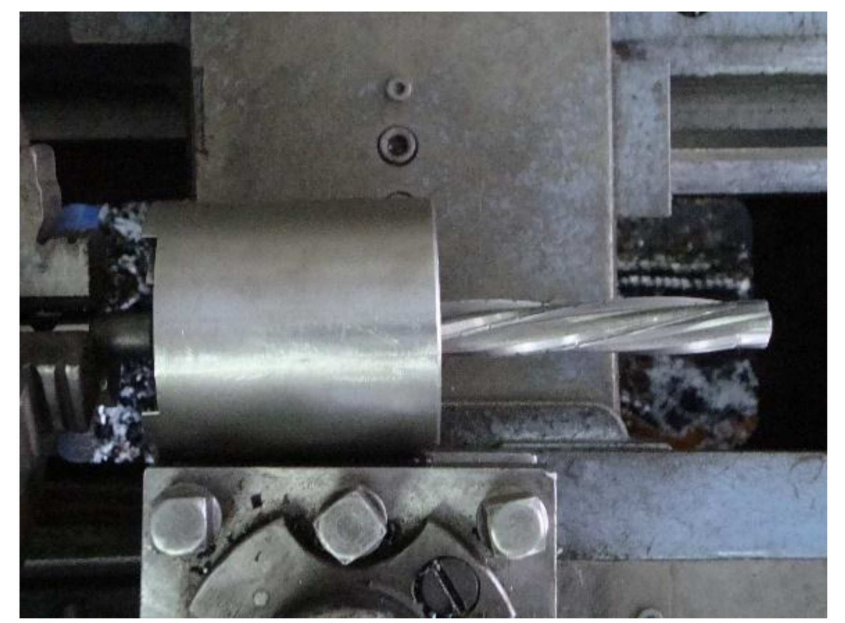

Figure 13. Free position of a hole-enlarging multiflute drill-broach.

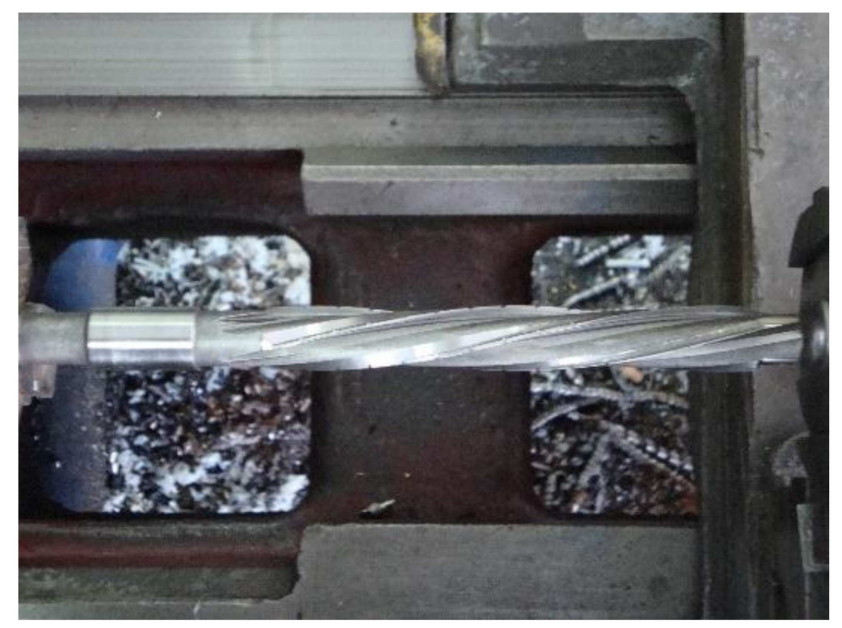

Figure 14. Terminal position of a hole-enlarging multiflute drill-broach.
Conducting experiments are associated with the determination of the minimum necessary, but sufficient number of experiments. To solve this problem, the mathematical apparatus of full-factorial experiment is applied by type $2^{3}$ [23].

The procedure for conducting experiments has been selected to be able to estimate the random errors of the experiment and avoid the effect of possible systematic errors. Identifying accidentally interfering factors, whose effect may be systematic, allowed the principle of randomization, which is used in the implementation of the matrix for experimental design.

To assess the reproducibility of experiment $G$, Cochran's $C$ test was used [24]. The experiment is reproducible, as $G_{\max }=0.537<G_{t a b}=0.7679$.

The experimental data were processed with the Excel program.

The adequacy assessment of the obtained regression equations was performed using Fisher's exact test $\left(F=1.441<F_{T}=5.32\right)$.

By transforming the regression equation, empirical relationships were derived:

1. The surface roughness:

$$
R_{a}=0.1487+0.0004 n-0.109 S .
$$

2. The depth of the surface defect layer:

$$
h=1.15+0.032 n-11.364 S \text {. }
$$

3. Surface hardness:

$$
H B=126.3+0.099 n-60 S .
$$

4. The diameter deviation:

$$
\delta=19.793+0.000186 n-0.0455 S
$$

\section{Results and discussions}

The main characteristic of the multi-flute drill-broach is a compound of the multi-flute drill and the broach features in one tool. This factor improves cutting conditions.

The results of the samples testing treated with the new cutting tool, i.e., hole-enlarging multi-flute drillbroach, and a new way of processing are as follows:

- The accuracy of the diametrical size of the holes is $0.018 \cdots 0.033 \mathrm{~mm}$ (7-8 IT Grade);

- The surface roughness of the holes is in the range of $R_{a}=0.16 \cdots 0.32 \mu \mathrm{m}$, which corresponds to 9 and 10 grades of roughness.

Analysis of the results shows that the precision of holes diametric sizes after the treatment with holeenlarging multi-flute drill-broach increased by 1,2 IT 
Grade as compared with the quality of cylindrical holeenlarging multi-flute drill; roughness decreased by 2 and 3 grades. In this case, feed per tooth if necessary can be reduced, and total allowance for processing holeenlarging multi-flute drill-broach is reduced.

According to the results of the data, the graphs represent the speed effect and feed on the breakdown of the holes diameter with hole-enlarging multi-flute drillbroach. Figures 15 and 16 are graphs of the precision of diametric dimensions of speed and feed to handle openings diameter $19.8 \mathrm{~mm}$.

As can be seen from the graphs, the diametrical size deviation increases with rotation frequency and decreases with increasing feed. This is due to the fact that the processing holes with the multi-flute drillbroach are close to broaching process.

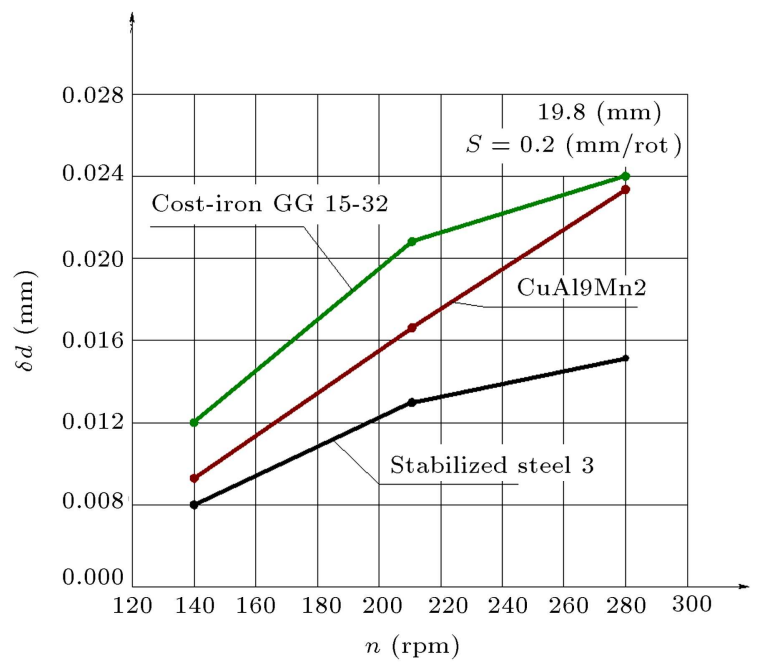

Figure 15. Graph of diametrical size accuracy dependence on the rotation frequency for processing diameter $19.8 \mathrm{~mm}$.

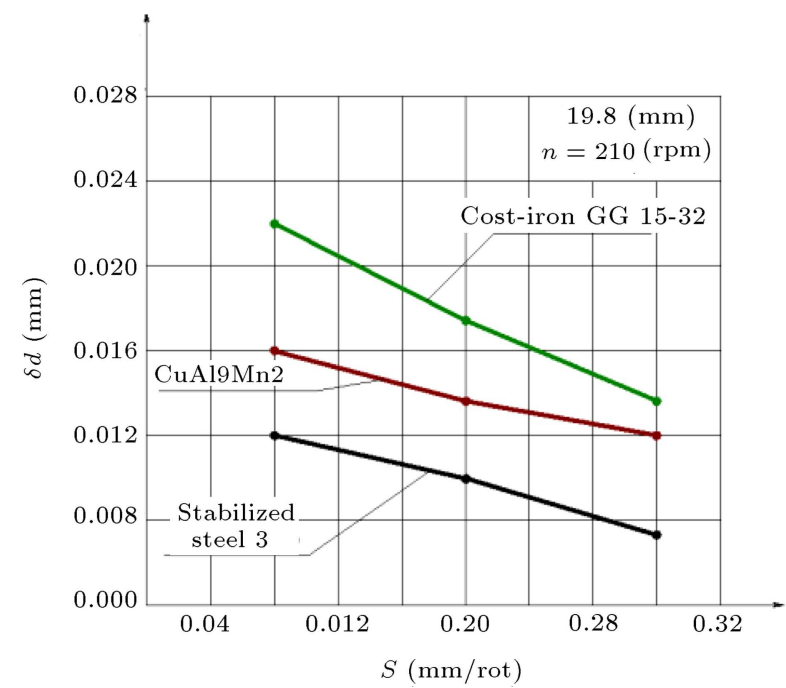

Figure 16. Graph of diametrical size accuracy dependence on the feed for processing $\varnothing 19.8 \mathrm{~mm}$.
The surface roughness of holes was measured on a profilometer 259 by the contact method.

Figures 17 and 18 represent graphs of the roughness dependence on rotation frequency of feed for the treatment of holes $\varnothing 19.8 \mathrm{~mm}$.

The proposed approach makes it possible to radically solve many problems of tool wear because the geometry of the proposed instrument and, consequently, forces and cutting forces contribute to less intense conditions for the operation and performance of instruments. In other words, the tool geometry allows, based on already known and proven structural materials, to provide tools with greater service life. Moreover, due to better distribution of forces in the tool; beats reduce drastically and results improve quality of material treatment, reduce roughness, and improve alignment holes complex profile of processing quality in general.

After machining with a hole-enlarging multiflute drill-broach, the hole surface microstructure was determined according to the procedure. The pol-

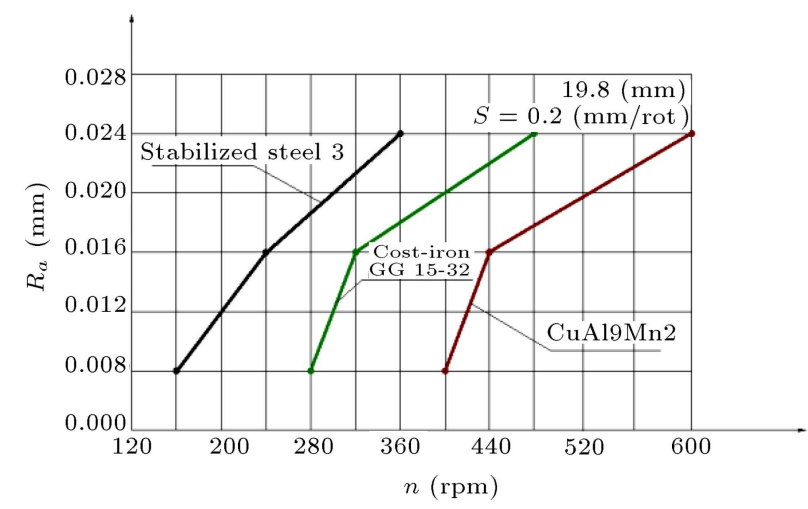

Figure 17. Graph of the roughness dependence on the speed machining of holes $\varnothing 19.8 \mathrm{~mm}$.

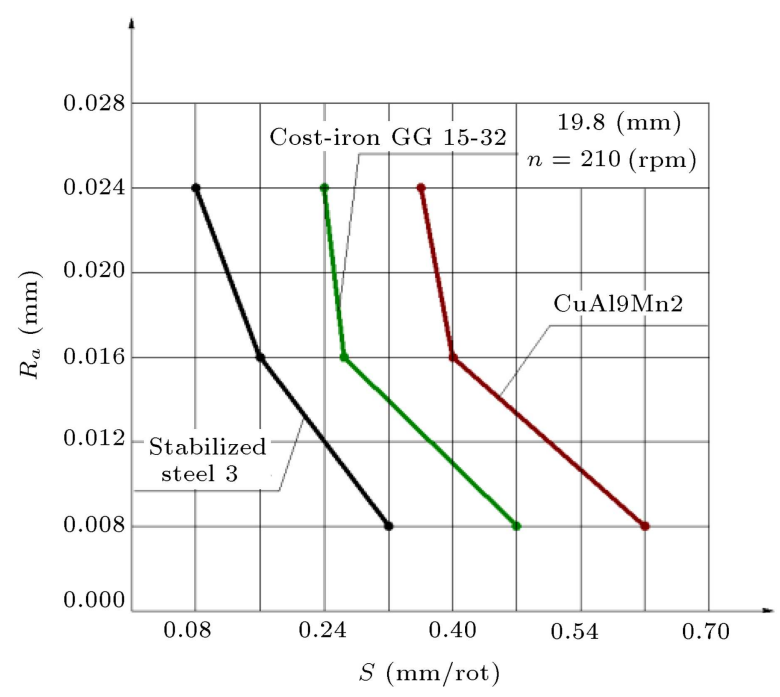

Figure 18. Graph of the roughness dependence on the feed in the processing holes $\varnothing 19.8 \mathrm{~mm}$. 


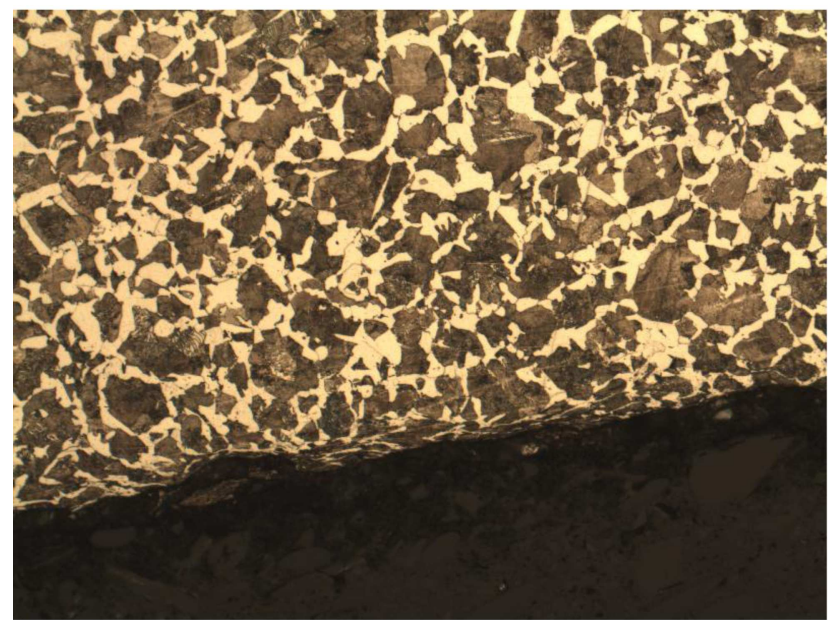

Figure 19. Microstructure of a sample no. 4, machined with a hole-enlarging multiflute drill-broach $\times 200$.

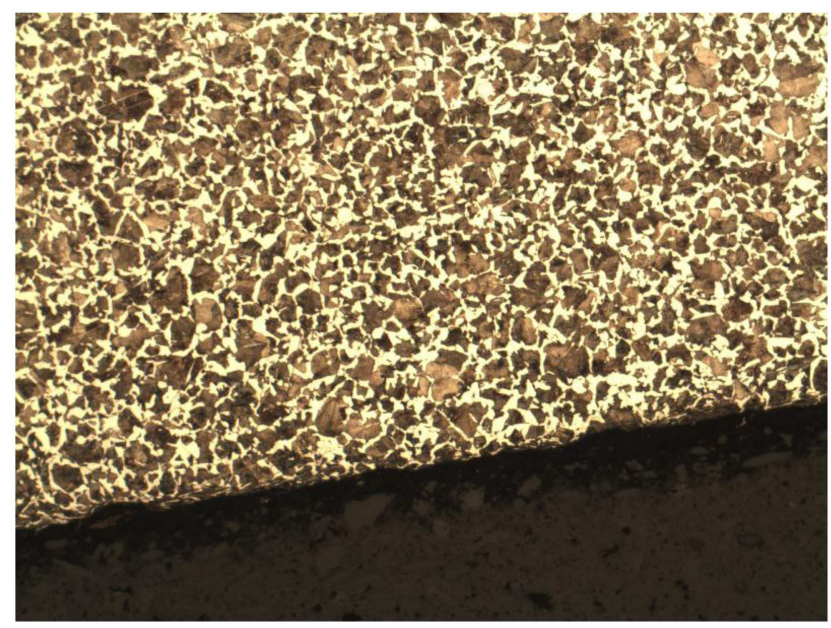

Figure 20. Microstructure of a sample no. 4, machined with a hole-enlarging multi-flute drill-broach $\times 100$.

ished section was studied with a OLYMPUS BX2M metallographic microscope for surface microstructures (Figures 19 and 20).

\section{Conclusion}

The quality and productivity of the holes machining process heavily depend on the tool design, which defines the cutting conditions. A procedure is described for an optimal design of the new multi-flute drill-broach tool.

To sum up, experimental studies show that the processing of holes with the multi-flute drill-broach provides higher quality of holes surface, decreases roughness, depth of the defect layer, a breakdown of the holes. This is due to benign cutting conditions in comparison with standard multi-flute drill. Due to the taper cutting part of the multiflute drill-broach, a constant pulling force eliminates the cutting process's continuity influence, reduces the overall length of the simultaneous cutting edges of the teeth; moreover, as a consequence, the tool life has increased by 2-3 times compared to a standard multi-flute drill.

Analysis of the results shows that the diametrical size accuracy of holes after the treatment with the multi-flute drill-broach has increased by 1 and 2 quality grade compared to broaching; roughness has decreased by 1 and 2 grade.

Analysis of the above graphs allows for optimal selection of processing modes for high-precision parts (accuracy of geometric dimensions, the minimum roughness is provided at relatively low speeds and high feed rates, and the depth of the defective layer increases with increasing speed and decreases with increasing tool feed).

Microstructure analysis of samples, machined with a hole-enlarging multiflute drill-broach, showed that inner surface has areas with a deformed layer, whose maximum depth is $25 \mu \mathrm{m}$. This is a ferritepearlite microstructure.

\section{Acknowledgements}

This study is part of a grant under the Young Scientist Program of the S. Toraighyrov Pavlodar State University on topic no. C1 "Determination and designation of cutting modes for machining holes with new cutting tools" order no. 1-02-07/683 dated 11/23/2009. Research continues to date within the initiative-search topic "Research and development of resource-energysaving metal-cutting tools" from its own funds, which is registered in the National Center of Science and Technology Evaluation JSC (Kazakhstan) registration number 0117PKU0382, 2017.

\section{References}

1. Webzell, S. "Analysis of factors affecting the tool life", Metalwork. Prod., 150, pp. 65-66 (2006).

2. Wang, Y.J., Zhang, D.H., Wu, F.J., Yao, K., and Hou, Z.M. "Simulation of cutting force based on software deform ICICTA: 2009", In Second Int. Conf. Intell. Comput. Technol. Autom., pp. 224-227 (2009).

3. Vogtel, P., Klocke, F., Lung, D., and Terzi, S. "Automatic broaching tool design by technological and geometrical optimization", Procedia CIRP, 33, pp. 496-501 (2015).

4. Vogtel, P., Klocke, F., Puls, H., Buchkremer, S., and Lung, D. "Modelling of process forces in broaching inconel 718", Procedia CIRP, 8, pp. 409-414 (2013).

5. Goncalves, D.A. and Schroeter, R.B. "Modeling and simulation of the geometry and forces associated with the helical broaching process", Int. J. Adv. Manuf. Technol., 83, pp. 205-215 (2016). 
6. Hosseini, A. and Kishawy, H.A. "Prediction of cutting forces in broaching operation", J. Adv. Manuf. Syst., 12(01), pp. 1-14 (2013).

7. Kishawy, H.A., Hosseini, A., Moetakef-Imani, B., and Astakhov, V.P. "An energy based analysis of broaching operation: Cutting forces and resultant surface integrity", CIRP Ann. - Manuf. Technol., 61, pp. 107110 (2012).

8. Klocke, F., Gierlings, S., Brockmann, M., and Veselovac, D. "Force-based temperature modeling for surface integrity prediction in broaching nickel-based alloys", Procedia CIRP, 13, pp. 314-319 (2014).

9. Lazarev, D.E. and Nasad, T.G. "Cutting tools for improving the quality and productivity of precise holes machining", Mach. Tools., 1, pp. 14-17 (2014).

10. Dudak, N.S., Itybaeva, G.T., Kassenov, A.Z., and Mussina, Z.K. "Part of the 14th Nechniké Sciences", In Proc. IV Int. Sci. - Pract. Conf. "Scientific Ind. Eur. Cont. - 2008", Publishing House <<Education and Science $>>$, Prague, pp. 67-71 (2008).

11. Beju, L.D., Brîndaşu, D.P., Muţiu, N.C., and Rothmund, J. "Modeling, simulation and manufacturing of drill flutes", Int. J. Adv. Manuf. Technol, 83(9-12), pp. 2111-2127 (2016).

12. Gupta, K.K., Jain, T., and Deshmukh, M. "Optimization of process parameters in high rpm micro drilling machine", Int. J. Innov. Eng. Technol., 2, pp. 128-130 (2013).

13. Zhao, C., Liang, Z., Zhou, H., and Qin, H. "Investigation on shaping machining method for deep hole keyway based on on-line symmetry detection and compensation", Journal of Mechanical Science and Technology, 31(3), pp. 1373-1381 (2017).

14. Kwon, K.B., Song, C.H., Park, J.Y., Oh, J.Y., Lee, J.W., and Cho, J.W. "Evaluation of drilling efficiency by percussion testing of a drill bit with new button arrangement", International Journal of Precision Engineering and Manufacturing, 15(6), pp. 1063-1068 (2014).

15. Bulat, P. and Volkov, K. "Detonation jet engine. Part 1 - Thermodynamic cycle", Int. J. Environ. Sci. Educ., 11, pp. 5009-5019 (2016).

16. Bulat, P., Volkov, K., and Ilyina, T. "Interaction of a shock wave with a cloud of particles", IEJMEMathematics Educ., 11, pp. 2949-2962 (2016).

17. Baroiu, N., Berbinschi, S., Teodor, V., and Oancea, $\mathrm{N}$. "The modeling of the active surfaces of a multiflute helical drill with curved cutting edge using the SV\& Toolbox environment", in: Proc. 13th Int. Conf. Tools-ICT, Miskolc, pp. 259-264 (2012).
18. Baroiu, N., Boazu, D., Vasilache, C.A., and Teodor, V. "Modeling of stress in drills with curved cutting edges", Appl. Mech. Mater., 371, pp. 509-513 (2013).

19. Lorincz, J. "Using digital tools to optimize your cutting tools", Manuf. Eng. Mag., 152(1), pp. 55-62 (2014).

20. Dudak, N.S., Kasenov, A.Z., Musina, Z.K., Itybaeva, G.T., and Taskarina, A.Z. "Hole-making with the use of reaming and broaching tool", Life Sci. J., 11, pp. 282-288 (2014).

21. Byrne, G. "Current state of various materials cutting technology and its practical application area", Ann. CIRP., 52(2), pp. 483-507 (2003).

22. Benes, J. "Overview of cutting tools", Am. Mach., 6, pp. 18-20 (2007).

23. Fisher, R. "The Arrangement of field experiments", $J$. Minist. Agric. Gt. Britain., 33, pp. 503-513 (1926).

24. Cochran, W.G. "The distribution of the largest of a set of estimated variances as a fraction of their total", Ann. Hum. Genet., 11, pp. 47-52 (1941).

\section{Biographies}

Nikolay Dudak has been a PhD candidate of Engineering Sciences. Now, he is a Professor at the Mechanical Engineering and Standardization Department in S. Toraighyrov Pavlodar State University.

His main works was "TRUE LIC N.K. REERH", which was printed in Pavlodar, S. Toraighyrov. Also, in co-authorship with Janushkin, As in 2011, he wrote next work "Method and cutter head for highperformance face performance of holes". Besides, he has three self-written works "Cosmic morality, the cosmic knowledge of unity and brotherhood", "A true world view is the epistemological basis of secular education of morality" and "Thin-energy-information Basis and Beauty of Life and Evolution".

Galiya Itybaeva has been a $\mathrm{PhD}$ candidate of Engineering Sciences. Now, she is an Associate Professor at the Mechanical Engineering and Standardization Department in S. Toraighyrov Pavlodar State University.

Her main works were written in co-authorship and called "Processing of holes with a reamer-broach" (2014) and "Hole machining based on using an incisive built-up reamer" (in 2017).

Asylbek Kasenov has been a $\mathrm{PhD}$ candidate of Engineering Sciences. Now, he is an Associate Professor at the Mechanical Engineering and Standardization Department in S. Toraighyrov Pavlodar State University.

His main works were written in co-authorship and called "Control holes with calibers" (in 2013); "Review and analysis of the capabilities of application programs" (in 2017); "Processing of holes with a reamer-broach" (in 2014); "An issue of intelligent 
road transport in Kazakhstan" (in 2017); and "Hole machining based on using an incisive built-up reamer" (also in 2017).

Zhanara Mussina has been a PhD candidate of Engineering Sciences. Now, she is an Associate Professor at the Mechanical Engineering and Standardization Department in S. Toraighyrov Pavlodar State University.

Her main works were written in co-authorship and called "The use of magnetic phenomena in nondestructive control" and "Hole machining based on using an incisive built-up reamer".

Aizhan Taskarina holds a PhD degree and works as an Associate Professor at the Metallurgy Department in S. Toraighyrov Pavlodar State University.

Her main works were written in co-authorship and called "Physical phenomena in the tool zone during the hole-making operations of the tool slide built-up reamer" (in 2014); "The method of finishing turning billets with an inclined axis through a continuous saber cutter" (in 2014); "The design of broaching profile cutting with screw-like equal teeth" (in 2014); "International scientific conference of young scientists, undergraduates, students and schoolchildren "XV Satpayev readings"" (in 2015) and "Materials of the $X$ international scientific and practical conference "Conduct of modern science-2014" (in 2014).

Kairatolla Abishev has been a PhD candidate of Engineering Sciences. Now, he is an Associate Professor at the Transport Equipment and Logistic Department in S. Toraighyrov Pavlodar State University. 